\title{
Prevalência de distúrbios osteomusculares relacionados ao trabalho de extrativismo
}

\section{mineral}

\author{
Prevalence of work-related musculloskeletal disorders in mineral extraction \\ Prevalencia de los trastornos musculoesqueléticos relacionados con el trabajo en la extracción de \\ minerales
}

Recebido: 15/01/2022 | Revisado: 23/01/2022 | Aceito: 26/01/2022 | Publicado: 27/01/2022

Danielle Cruz da Luz

ORCID: https://orcid.org/0000-0002-1734-0613 Universidade Federal do Maranhão, Brasil E-mail: daniellecruzdaluz@hotmail.com

Keyla Cristina Nogueira Durans

ORCID: https://orcid.org/0000-0003-4963-5448 Universidade Federal do Maranhão, Brasil E-mail: Keyla.durans@discente.ufma.br Adriano Constância de Araújo

ORCID: https://orcid.org/0000-0002-1468-1404

Universidade Federal do Maranhão, Brasil E-mail: adrianoaraujoenf@gmail.com

Rosely Lopes Figueredo

ORCID: https://orcid.org/0000-0002-1235-2172 Universidade Federal do Maranhão, Brasil E-mail: litazelita@gmail.com

Maria do Carmo Lacerda Barbosa ORCID: https://orcid.org/0000-0001-7265-8627 Universidade Federal do Maranhão, Brasil Fundação Oswaldo Cruz, Brasil E-mail: carminha13032009@hotmail.com

Maria Raimunda Santos Garcia ORCID: https://orcid.org/0000-0002-5355-5654 Universidade Federal do Maranhão, Brasil E-mail:mrs.garcia@ufma.br

\begin{abstract}
Resumo
Objetivo: estimar a prevalência de sintomas de distúrbios musculoesqueléticos em trabalhadores do extrativismo mineral. Método: Estudo de natureza quantitativa, descritiva e transversal que foi realizado no povoado de Pirinã, Município de Pinheiro, no estado do Maranhão. O universo da pesquisa compreendeu os trabalhadores do extrativismo de pedra. A amostra foi de conveniência por inclusão de indivíduos que apresentavam sintomatologia dolorosa em alguma região anatômica do corpo. A coleta dos dados foi feita mediante o uso de dois instrumentos, o Questionário Individual (QI) e o Questionário Nórdico de Sintomas Musculoesquelético (QNSM). Resultados: A maioria apresentou dor na coluna lombar, coluna dorsal, mãos/punhos, e ombros, necessitando de afastamento do trabalho. Em relação ao cansaço físico durante a jornada de trabalho, $80 \%$ referiram cansaço ao final da jornada de trabalho observou-se também que 23 trabalhadores possuíam mais de 10 anos de trabalho e laboravam de 3-4 horas por dia durante a semana. Considerações finais: Os resultados chamam a atenção quanto a recorrência da dor entre os trabalhadores rurais, dores estas que podem interferir em sua qualidade vida, causar sequelas irreversíveis e/ou crônicas e diminuir sua produtividade. A análise ergonômica neste contexto pode atuar prevenindo a incidência de acidentes e evitando incapacitações.
\end{abstract}

Palavras-chave: Ergonomia; Distúrbios musculoesqueléticas; Saúde do trabalhador rural; Mineradores.

\begin{abstract}
Objective: to estimate the prevalence of musculoskeletal disorders symptoms in mineral extraction workers. Method: A quantitative, descriptive, and cross-sectional study was conducted in the village of Pirinã, Municipality of Pinheiro, Maranhão State. The research universe comprised the workers of stone extraction. The sample was of convenience for the inclusion of individuals who presented painful symptoms in some anatomical region of the body. Data collection was done through the use of two instruments, the Individual Questionnaire (IQ) and the Nordic Musculoskeletal Symptoms Questionnaire (NSQ). Results: The majority presented pain in the lumbar spine, dorsal column, hands/wrists, and shoulders, requiring time off work. In relation to physical tiredness during the workday, $80 \%$ reported tiredness at the end of the workday. It was also observed that 23 workers had worked for more than 10 years and worked 3-4 hours
\end{abstract}


a day during the week. Final considerations: The results draw attention to the recurrence of pain among rural workers, which can interfere with their quality of life, cause irreversible and/or chronic sequels, and decrease their productivity. The ergonomic analysis in this context can act preventing the incidence of accidents and avoiding disabilities.

Keywords: Ergonomics; Musculoskeletal disorders; Rural worker's health; Miners.

\section{Resumen}

Objetivo: Estimar la prevalencia de los síntomas de los trastornos musculoesqueléticos en los trabajadores de la extracción de minerales. Método: Se realizó un estudio cuantitativo, descriptivo y transversal en la aldea de Pirinã, Municipio de Pinheiro, Estado de Maranhão, Brasil. El universo de la investigación estaba formado por los trabajadores de la extracción de piedra. La muestra fue de conveniencia para incluir a los individuos que presentaban síntomas dolorosos en alguna región anatómica del cuerpo. La recogida de datos se realizó mediante el uso de dos instrumentos, el Cuestionario Individual (CI) y el Cuestionario Nórdico de Síntomas Musculoesqueléticos (NSQ). Resultados: La mayoría presentaba dolor en la columna lumbar, la columna dorsal, las manos/muñecas y los hombros, lo que requería una baja laboral. En cuanto al cansancio físico durante la jornada laboral, el 80\% declaró estar cansado al final de la misma. También se observó que 23 trabajadores llevaban más de 10 años trabajando y trabajaban 3-4 horas al día durante la semana. Consideraciones finales: Los resultados llaman la atención sobre la recurrencia del dolor entre los trabajadores rurales, que puede interferir en su calidad de vida, causar secuelas irreversibles y/o crónicas y reducir su productividad. El análisis ergonómico en este contexto puede ayudar a prevenir la incidencia de accidentes y a evitar las incapacidades.

Palabras clave: Ergonomía; Trastornos musculoesqueléticos; Salud de los trabajadores rurales; Mineros.

\section{Introdução}

Os processos de saúde e doença, em uma perspectiva integral, apresentam grande dinamicidade. Eles podem estar diretamente articulados com determinantes e condicionantes de saúde como meios de subsistência e de produtividade, ao decorrer de toda a vida de uma população (Silva \& Mota, 2019).

Desta forma, saúde e Qualidade de Vida (QV) são conceitos indissociáveis, pois uma melhor QV não se resumirá em somente melhores condições financeiras e/ou materiais, mas também em um entorno ambiental agradável que proporcione ao indivíduo sua autonomia e possibilidade de realização pessoal e profissional. Entretanto, em meio às atividades desenvolvidas, o organismo do indivíduo pode reagir de maneira inesperada, quando exposto a grande carga de trabalho. Por esta razão é crescente a quantidade de pesquisas que buscam estabelecer a relação entre as atividades desenvolvidas e a instauração de complicações musculoesqueléticas, sobretudo em ambiente laboral (Duarte \& Cavaignac, 2019; Paula \& Amaral, 2019; Zavarizzi et al., 2019)

Os Distúrbios Osteomusculares Relacionados ao Trabalho (DORT) ocorrem quando há sobrecarga de força ou de pressão, bem como movimentos repetitivos sobre determinados segmentos corporais, associadas a ausência de tempo hábil para sua recuperação. Os sintomas relacionados ao agravo são dores, fadiga, sensação de peso nas áreas acometidas e parestesias, podendo ser localizados ou difusos, determinando o afastamento do trabalhador da sua rotina laboral, perdas financeiras, redução da capacidade laboral parcial ou total e da sua autonomia para desempenhar atividades da vida diária (Rodrigues et al., 2020; Silva et al., 2020).

A Ergonomia é a ciência que estuda a relação entre as pessoas em seu espaço de trabalho, equipamentos e ambiente, com especial relação ao conjunto de interferências e impactos anatômicos, fisiológicos e psicológicos ao indivíduo no desempenho da sua atividade produtiva (Lopes, 2017). As inadequações ergonômicas e o desenvolvimento de incapacidade laboral, advindas da exposição a fatores de risco, muita das vezes evitáveis, é responsável pela instalação de um estado sofrimento contínuo ao trabalhador (Bernardo et al., 2017; Santos, 2018).

O trabalho com extração mineral é considerado pelo Organização Internacional do Trabalho (OIT) como uma das áreas mais perigosas de labor, recebendo classificação de risco IV, pois expõe os trabalhadores aos mais diversos riscos, como físicos, químicos, biológicos e sobretudo os ergonômicos, em virtude da falta de condições sanitárias e de local adequado para desenvolver sua atividade (OIT, 2002). As constantes atividades desempenhadas pela quebra e transporte de minérios demandam 
constantes esforços físicos e podem promover alterações importantes nas forças mecânicas desempenhadas pelos músculos, articulações e esqueleto, favorecendo maior esforço cardiovascular e favorecendo lombalgias (Minette et al., 2018).

Portanto, descrever as principais manifestações ergonômicas dos trabalhadores do extrativismo mineral é relevante, considerando o alto risco de doenças ocupacionais a qual estão continuamente expostos. Os resultados dessa pesquisa contribuirão para o desenvolvimento de novos equipamentos de proteção individual e coletiva, readequação ergonômica e prevenção de riscos melhorando continuamente a qualidade de vida, reduzindo custos sociais e previdenciários.

\section{Metodologia}

Estudo de natureza quantitativa, descritiva e transversal que foi realizado no povoado de Pirinã, Município de Pinheiro, no estado do Maranhão, durante o período de junho a agosto de 2020.

O universo da pesquisa compreendeu os trabalhadores do extrativismo de pedra. A amostra foi de conveniência por inclusão de indivíduos que apresentavam sintomatologia dolorosa. Posteriormente, os pesquisadores procederam a seleção dos indivíduos que foram convidados a participar da pesquisa, obedecendo aos critérios de inclusão: trabalhadores maiores de 18 anos, ser portador de alguma manifestação musculoesquelética e que assinaram o Termo de Consentimento. Foram considerados como critérios de não inclusão: ter abaixo de 18 anos, não ser portador de manifestações musculoesqueléticas e indivíduos que não preencheram o TCLE.

A coleta dos dados foi feita mediante o uso de dois instrumentos, o Questionário Individual (QI) e o Questionário Nórdico de Sintomas Musculoesquelético (QNSM), proposto por Pinheiro et al. (2002). O QI apresentava questões relativas aos aspectos sociodemográficos dos participantes, presença de doenças crônicas e hábitos de vida, tempo e jornada de trabalho no extrativismo e avaliação subjetiva de saúde atual e pregressa.

Já o QNSM foi assinalado em forma de escolhas múltiplas ou binárias, com relação aos sintomas de desconforto ou dores em regiões como pescoço, ombro, parte superior das costas, cotovelos, parte inferior das costas, punhos e mãos, quadril e coxas, joelhos, tornozelos e pés, ilustradas por figura no canto superior do instrumento, possibilitando a identificação de sintomas musculoesqueléticos pelo trabalhador, a interferência na realização das atividades laborais, assim como a necessidade de procura por serviços de saúde.

Esta pesquisa foi aprovada pelo Comitê de Ética em Pesquisa (CEP), mediante parecer de número 4.043.096 e CAAE número 30530420.1.0000.5087. Todos os participantes da pesquisa preencheram o TCLE.

Os dados obtidos foram analisados e apresentados por meio de estatísticas descritivas, tabulados através da frequência e porcentagem do Programa SPSS (Statistical Package for the Social Sciences), versão 2.1 para Windows.

\section{Resultados}

Ao todo, participaram da pesquisa 40 trabalhadores, a maioria era do sexo feminino $(n=28)$, faixa etária entre 20-40 $\operatorname{anos}(n=27)$ grau de escolaridade fundamental incompleto $(n=17)$. Em relação a presença de comorbidades, a maioria dos participantes afirmou ter "outras doenças" $(\mathrm{n}=25)$ e em segundo lugar, serem portadores de hipertensão arterial (n=12). Quanto aos hábitos de vida, a maioria informou fazer uso de bebidas alcoólicas $(n=36)$ e destes $58 \%$ uso frequente. Em relação ao uso do tabaco, 21 afirmaram fazer uso, sendo que a maioria $(61,9 \%)$ fazia uso ocasional (Tabela 1).

Em relação ao cansaço físico durante a jornada de trabalho, a maioria $80 \%(n=32)$ referiram cansaço ao final da jornada de trabalho. Observou-se também que os trabalhadores possuíam mais de 10 anos de trabalho (n=23) 57,5\% e uma jornada de 3-4 horas/dia todos os dias da semana. Por fim, em relação ao questionamento sobre a qualidade de vida atual, a maior parte dos entrevistados considerava sua vida boa $(57,5 \%)$, mas se comparada há um ano, apontam que se mantém inalterada $(67,5 \%)$, conforme a Tabela 1 . 
Tabela 1 - Caracterização sociodemográfica, doenças crônicas, hábitos de vida, cansaço físico durante a jornada de trabalho e avaliação subjetiva de saúde dos trabalhadores do extrativismo mineral do povoado Pirinã $(\mathrm{n}=40)$.

\begin{tabular}{|c|c|c|c|c|c|}
\hline Variáveis & Total & $(\%)$ & & Total & $(\%)$ \\
\hline Sexo & & & Cansaço Físico na jornada de trabalho & & \\
\hline Masculino & 12 & 30 & Início da jornada & 0 & 0 \\
\hline Feminino & 28 & 70 & Durante a jornada & 7 & 17,5 \\
\hline Faixa Etária & & & Final da jornada & 32 & 80 \\
\hline Menor que 20 & 2 & 5 & Não se sente cansado & 1 & 2,5 \\
\hline 20 a 30 & 14 & 35 & & & \\
\hline 31 a 40 & 13 & 32,5 & Tempo de trabalho em anos & & \\
\hline 41 a 50 & 5 & 12,5 & $0-10$ anos & 13 & 32,5 \\
\hline 51 a 60 & 5 & 12,5 & $11-20$ anos & 11 & 27,5 \\
\hline Acima de 60 & 1 & 2,5 & $21-30$ anos & 11 & 27,5 \\
\hline Escolaridade no Brasil & & & Mais de 31 anos & 12,5 & 12,5 \\
\hline Fundamental incompleto ( $<9$ anos de estudo) & 17 & 42 & Tempo de trabalho em horas/dias & & \\
\hline Fundamental completo ( 9 anos de estudo) & 3 & 7,5 & $0-2$ horas/dia & 0 & 0 \\
\hline Ensino médio incompleto ( $<3$ anos de estudo) & 13 & 32,5 & 3-4 horas/dia & 36 & 90 \\
\hline Ensino médio completo ( 3 anos de estudo) & 7 & 17,5 & 5-6 horas/dia & 4 & 10 \\
\hline Superior incompleto & 0 & 0 & & & \\
\hline Superior completo & 0 & 0 & Classificação Atual da saúde dos Trabalhadores & & \\
\hline Pós-graduação & 0 & 0 & Excelente & 0 & 0 \\
\hline Presença de doença crônica & & & Muito boa & 1 & 2,5 \\
\hline Hipertensão & 12 & 30 & Boa & 23 & 57,5 \\
\hline Reumatismo & 2 & 5 & Ruim & 16 & 40 \\
\hline Diabetes & 3 & 7,5 & Muito ruim & 0 & 0 \\
\hline Doenças do coração & 0 & 0 & & & \\
\hline Depressão & 0 & 0 & Classificação da saúde em relação há um ano & & \\
\hline Câncer & 0 & 0 & Muito melhor & 0 & 0 \\
\hline Outras & 23 & 57,5 & Um pouco melhor & 0 & 0 \\
\hline Tabagismo & & & Quase a mesma coisa & 27 & 67,5 \\
\hline Sempre & 8 & 20 & Um pouco pior & 13 & 32,5 \\
\hline As vezes & 12 & 30 & Muito pior & 0 & 0 \\
\hline Raramente & 1 & 2,5 & & & \\
\hline Nunca & 19 & 47,5 & & & \\
\hline \multicolumn{6}{|l|}{ Etilismo } \\
\hline Sempre & 21 & 52,5 & & & \\
\hline As vezes & 12 & 30 & & & \\
\hline Raramente & 3 & 7,5 & & & \\
\hline Nunca & 4 & 10 & & & \\
\hline
\end{tabular}

Fonte: Autores (2022).

Para a análise das condições ergonômicas relacionadas a rotina de trabalhado dos pesquisados, foi utilizado o QNSM. Os participantes foram perscrutados sobre a ocorrência de dores em diferentes segmentos corporais nos últimos 12 meses e, se os sintomas determinaram absenteísmo, mesmo que durante apenas um dia. Como resultados (Tabela 2 e Gráfico 1), a maioria apresentou dor na coluna lombar $(n=33)$, coluna dorsal $(n=32)$, mãos/punhos $(n=27)$ e ombros $(n=27)$, necessitando de afastamento do trabalho. 
Tabela 2 - Ocorrência de dores/desconfortos nos trabalhadores do extrativismo mineral do povoado Pirinã $(n=40)$.

\begin{tabular}{|c|c|c|c|c|c|}
\hline Variável & Total & $(\%)$ & Variável & Total & $(\%)$ \\
\hline Pescoço & & & Joelhos & & \\
\hline Desconforto nos últimos 12 meses & 13 & 32,5 & Desconforto nos últimos 12 meses & 18 & 45 \\
\hline Precisou afastar-se das atividades & 12 & 30,7 & Precisou afastar-se das atividades & 15 & 37,5 \\
\hline Ombros & & & Tornozelos/Pés & & \\
\hline Desconforto nos últimos 12 meses & & & Desconforto nos últimos 12 meses & 6 & 15 \\
\hline Nenhum & 10 & 25 & Precisou afastar-se das atividades & 5 & 12,5 \\
\hline Direito & 27 & 67,5 & & & \\
\hline Esquerdo & 0 & 0 & & & \\
\hline Ambos & 3 & 7,5 & & & \\
\hline Precisou afastar-se das atividades & 26 & 65 & & & \\
\hline \multicolumn{6}{|l|}{ Cotovelos } \\
\hline Nenhum & 31 & 77,5 & & & \\
\hline Direito & 8 & 20 & & & \\
\hline Esquerdo & 0 & 0 & & & \\
\hline Ambos & 1 & 2,5 & & & \\
\hline Precisou afastar-se das atividades & 8 & 20 & & & \\
\hline \multicolumn{6}{|l|}{$\begin{array}{l}\text { Mão/Punho } \\
\text { Desconforto nos últimos } 12 \text { meses }\end{array}$} \\
\hline Nenhum & 7 & 17,5 & & & \\
\hline Direito & 27 & 67,5 & & & \\
\hline Esquerdo & 0 & 0 & & & \\
\hline Ambos & 6 & 15 & & & \\
\hline Precisou afastar-se das atividades & 2 & 5 & & & \\
\hline \multicolumn{6}{|l|}{ Coluna Dorsal } \\
\hline Desconforto nos últimos 12 meses & 32 & 80 & & & \\
\hline Precisou afastar-se das atividades & 30 & 75 & & & \\
\hline \multicolumn{6}{|l|}{ Coluna Lombar } \\
\hline Desconforto nos últimos 12 meses & 33 & 82,5 & & & \\
\hline Precisou afastar-se das atividades & 30 & 75 & & & \\
\hline \multicolumn{6}{|l|}{ Quadris ou coxas } \\
\hline Desconforto nos últimos 12 meses & 19 & 47,5 & & & \\
\hline Precisou afastar-se das atividades & 13 & 32,5 & & & \\
\hline
\end{tabular}


Figura 1 - Principais causas de afastamentos dos trabalhadores do extrativismo mineral residentes do povoado Pirinã.

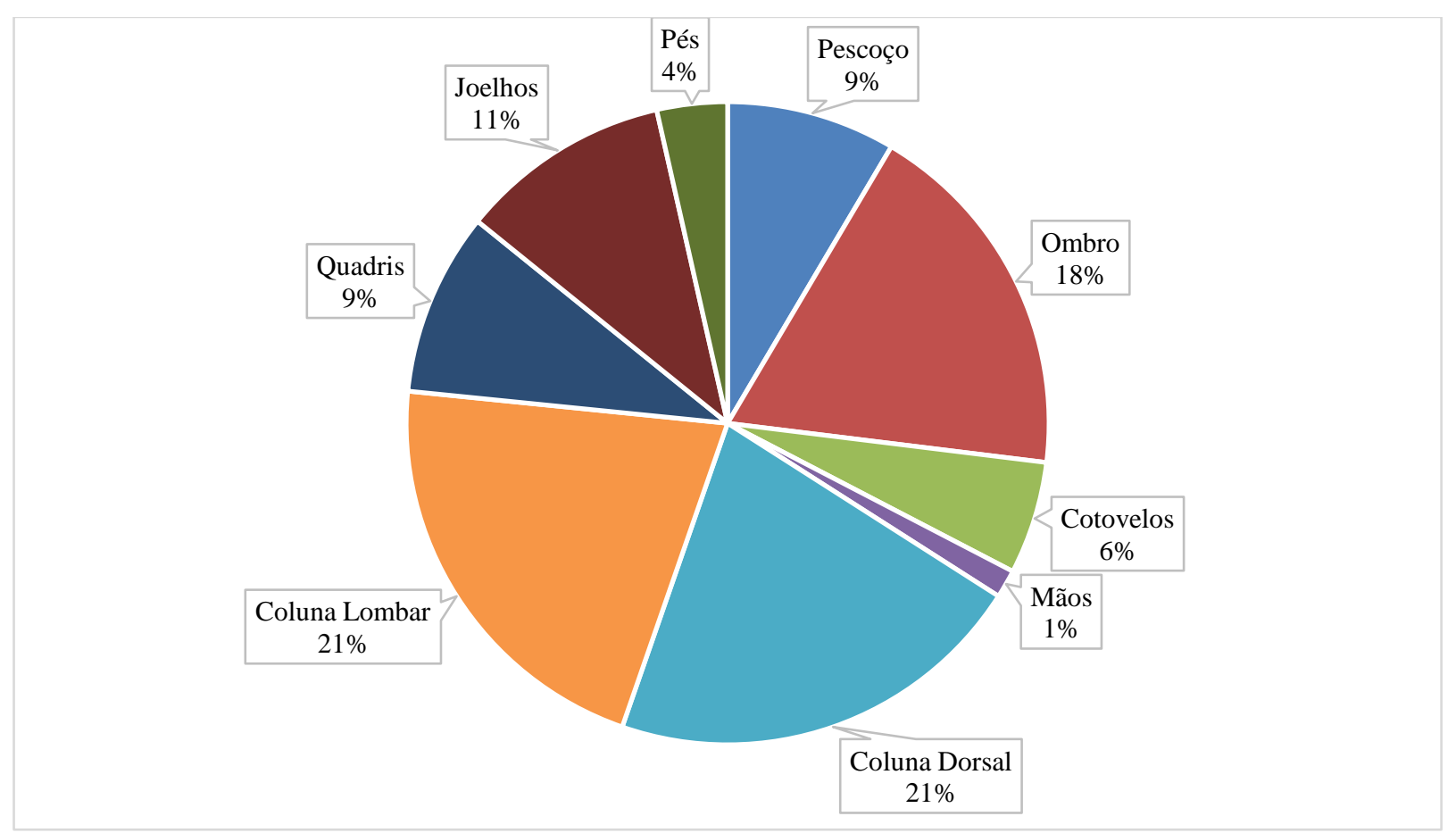

Fonte: Elaborada pelos autores a partir do QNSM (2022).

\section{Discussão}

O presente estudo estimou a prevalência de sintomas de distúrbios musculoesqueléticos em trabalhadores do extrativismo mineral no município de Pirinã - MA. Quarenta trabalhadores foram investigados, por amostra de conveniência, sendo este o primeiro estudo realizado nesta comunidade.

De forma semelhante a vários estudos com trabalhadores rurais, a exemplo das quebradeiras de coco babaçu analisadas por Vale et al. (2018) e Barbosa et al. (2015), o presente trabalho mostra que mulheres formam a maior parte da força de trabalho extrativista. A baixa escolaridade concorre para a dificuldade de acesso as informações e conhecimento das normas técnicas, além de que em função da estrutura muscular feminina concorre para maior risco de lesões musculotendíneas (Cardoso, 2021).

É comum a falta de conhecimento sobre a importância do uso Equipamentos de Proteção Individual (EPIs) aliado à ideia de que agregam maior peso e dificuldade no desenvolvimento das atividades. Destaca-se que a baixa escolaridade pode interferir em diversos aspectos de compreensão, raciocínio e cognição dos trabalhadores, resultando em baixa assimilação da linguagem técnica contida em manuais ou demais materiais que podem ser usados para ensiná-los sobre aspectos ergonômicos, e conhecimento sobre os riscos de acidentes e deficiências advindas do trabalho realizado de maneira inadequada (Schettino $e t$ al., 2020; Souza et al., 2021).

Neste estudo, houve uma maior ocorrência de doenças as quais os trabalhadores não conseguiram classificar, contudo de forma semelhantes a trabalhos realizados em populações quilombolas do Maranhão, a Hipertensão Arterial Sistêmica (HAS) foi muito frequente (Barbosa et al., 2015). As doenças cardiovasculares e metabólicas, a exemplo também da diabetes Mellitus tipo II tem raízes que perpassam por questões alimentares e relativas aos modos de viver, além da carga hereditária (Melo et al., 2020; Oliveira et al., 2008).

Estudos realizados por Mendes et al. (2019) corroboram com estes resultados, ao trazerem mais de 50\% dos participantes com alguma Doença Crônica Não Transmissível (DCNT) com associações a qualidade de vida de maneira abrangente, incluindo falta de acesso à informação, baixa escolaridade e necessidade de grande produtividade no trabalho, que proporciona pouco tempo para o autocuidado (Mendes et al., 2019; Victorio \& Gonçalves, 2021). 
A ocorrência de doenças cardíacas e metabólicas contribui para o agravamento de lesões em órgãos como rins, cérebro e arteriais, vitais ao nosso corpo (Dias, 2020). A presença da nicotina também pode contribuir ao desenvolvimento de outras DCNT, como o câncer, e a ingesta de mais de 6 dose diárias de álcool já foi apontada como fator contribuinte ao aumento do sódio sanguíneo e diminuição de efeitos das medicações contra a HAS (Klatsky, 2016).

O consumo de bebidas alcóolicas foi comum nos trabalhadores do extrativismo mineral nesta pesquisa, dado também encontrado em outros estudos com trabalhadores rurais (Barbosa et al., 2015, Melo et al., 2020).

O cansaço físico durante a jornada de trabalho foi apontado por $80 \%$ dos participantes da pesquisa. De acordo com Silva et al., (2020) e Ximenes Neto et al., (2020) este sintoma pode estar associado a permanência em posição fixa por longos períodos durante o dia que promove o uso dos mesmos grupos musculares e sua possível sobrecarga. Além disso, outras condições podem estar aliadas ao cansaço como a baixa ingestão hídrica, longos períodos de exposição ao calor e umidade, levantamento de materiais pesados e ausência de pausas durante o tempo trabalhado.

O tempo de trabalho em anos obtidos pela pesquisa demonstra características que indicam, que o trabalho em ambiente rural acontece desde a adolescência e perdura por toda a vida adulta, em seu ápice de produtividade, conforme aponta Mendes et al. (2019) e Ximenes Neto et al. (2020). Estes enfatizam que os modos de atuação são passados por gerações baseado em conhecimentos empíricos sem qualquer amparo técnico-profissional e a necessidades de subsistência faz com que o trabalhador se mantenha naquela atividade durante a fase avançada da vida.

A percepção da qualidade de vida de 57,5\% dos participantes foi classificada como boa, entretanto diversos autores chamam a atenção para a análise subjetiva deste significado. A percepção de qualidade de vida se insere em vários componentes essenciais como direito à moradia, emprego, convívio social, adequado estado de saúde e segurança, contudo é comum que o termo seja relacionado apenas com as condições de saúde. Não estar doente e conseguir ser produtivo são mencionados como pontos cruciais a uma elevada $\mathrm{QV}$, sendo assim, trabalhar e trazer o sustento à família seria fonte de alegria e satisfação. (Carvalho \& Santos, 2020; Miranda et al., 2020; Cardoso et al., 2021).

O afastamento dos trabalhadores em nosso estudo foi em sua maior parte, causado por dores na coluna (lombar e dorsal). As lombalgias têm sido cada vez mais estudadas por conta da grande taxa de absenteísmo e deficiências que podem ocasionar ao trabalhador. Como evidenciado por outros autores, suas raízes estão principalmente em movimentos repetitivos, sobrecarga de atividades, aumento da tensão por levamento excessivo de peso e ambiente inadequado de desenvolvimento de atividades laborais (Andrade et al., 2019; Silva et al., 2021; Silveira et al., 2020; Vale et al., 2018).

A ausência de conhecimento sobre o posicionamento corporal correto para abaixar-se e curvar o tronco durante o processo de extrativismo representa a principal causa de dor lombar nesses trabalhadores de acordo com Maia (2020) e Melek et al., (2017).

A sintomatologia dolorosa promove reflexos negativos na rotina do trabalhador. Considerando que nosso estudo foi composto em sua maioria por mulheres, é necessário observar que além das atividades trabalhistas, as funções dentro do lar também precisam ser realizadas, demandando esforço das estruturas no momento de descanso do trabalhador.

Em estudo realizado por Carvalho e Santos (2020), a quantidade de trabalhadores com dores na coluna, avaliados pelo QNSM foi de 21,2\%, sendo constatada associação estatística entre as dores e a escolaridade dos participantes. O sexo também foi apresentado como um fator, estando as mulheres mais expostas às incapacitações (Carvalho et al., 2020; Simas et al., 2020).

Para prevenção de tais agravos, faz-se necessário um olhar mais atento da equipe de saúde, buscando formas de introduzir a educação em saúde no desenvolvimento das atividades de maneira ergonomicamente correta, bem como proporcionar o (re)conhecimento da postura inadequada durante o desenvolvimento das funções na mineração (Silva et al., 2020). Ademais, cabe aos empregadores conhecer e apropriar-se das Normas Regulamentadoras (NR) 17, que trata sobre a 
adaptação das atividades aos parâmetros psicofisiológicos de cada trabalhador, e a NR 31 que estabelece que o empregador rural deverá oferecer capacitações frequentes aos seus subordinados, de maneira a prevenir incidentes evitáveis à saúde do trabalhador (Brasil, 2018; Veiga et al., 2017).

Como potencialidade deste estudo pode-se apontar a possibilidade de caracterização dos trabalhadores do extrativismo mineral, elucidando assim as causas mais comuns de afastamento de suas atividades laborais, bem como o baixo custo para realização da coleta de dados. Entretanto, como fragilidades estão a impossibilidade de acompanhamento por um maior período, haja visto que esta pesquisa teve caráter transversal, impossibilitando uma análise temporal da causa e efeito relacionados as dores e afastamento.

\section{Considerações Finais}

Os dados obtidos possibilitaram a caracterização sociodemográfica dos trabalhadores do extrativismo mineral do povoado Pirinã-MA e identificaram segmentos corporais mais acometidos por sintomatologia e agravos musculoesqueléticos. A maioria dos trabalhadores foram do sexo feminino, na faixa etária entre a segunda e a terceira década da vida; com grau de escolaridade abaixo de 9 anos de estudo. A Hipertensão arterial foi a doença identificada como a mais prevalente e o uso habitual de bebidos alcoólicas foi referido pela maioria dos participantes da pesquisa. Em relação às manifestações musculoesqueléticas, os segmentos corporais mais afetados em ordem decrescente foram colunar lombar, coluna dorsal, punhos e ombros, sendo causa de absenteísmo no trabalho.

Os resultados chamam a atenção quanto a recorrência da dor entre os trabalhadores rurais, dores estas que podem interferir em sua qualidade vida, causar sequelas irreversíveis e/ou crônicas e diminuir sua produtividade. A análise ergonômica neste contexto pode atuar prevenindo a incidência de acidentes e evitando incapacitações. Investimentos na Educação, são fundamentais para o acesso e compreensão das informações sobre as condições adequadas de trabalho e os seus direitos enquanto trabalhadores rurais.

É imprescindível a implementação de uma política de promoção a segurança no trabalho por parte dos empregadores, embasados nas NRs vigentes e, aliado a isso, uma assistência mais aproximada da equipe da Estratégia Saúde da Família (ESF) também atuante na área rural, embasada pela Política Nacional de Saúde Integral das Populações do Campo e da Floresta (PNSIPCF), contribuindo para a promoção de acesso a saúde de maneira humanizada, minimizando riscos provenientes dos determinantes e condicionantes de saúde.

\section{Referências}

Andrade, I. C. C., Freitas, C. K. A. C., Barreiro, M. do S. C., \& Menezes, A. F. de. (2019). Perfil demográfico e clínico de trabalhadores atendidos em um Centro de Referência em Saúde do Trabalhador. Revista Brasileira de Pesquisa em Saúde/Brazilian Journal of Health Research, 21(1), 28-34.

Barbosa, M. do C. L., Barbosa, J. B., Guerra, L. F. A., Barbosa, M. F. L., Barbosa, F. L., Barbosa, R. L., Guida, D. L., Martins, M. L. B., Bouskela, E., Nascimento, M. do D. S. B., Melo, G. S. de O., \& Castro, M. M. da S. (2015). Dislipidemia e risco cardiovascular em afrodescendentes: Um estudo em comunidades quilombolas do Maranhão, Brasil. Rev. bras. med. fam. comunidade, 1-10.

Bernardo, D. C. dos R., Nascimento, J. P. de B., Silveira, P. R. da, \& Soares, K. G. R. (2017). O estudo da ergonomia e seus benefícios no ambiente de trabalho: Uma pesquisa bibliográfica. Saberes Interdisciplinares, 6(11), 97-112.

BRASIL (2018). NR-31 - Segurança e Saúde no Trabalho na Agricultura, Pecuária Silvicultura, Exploração Florestal e Aquicultura. Portaria MTb n. 1.086 , de 18 de dezembro de 2018 .

Cardoso, L. S., Bonow, C. A., Xavier, D. M., \& Cezar-Vaz, M. R. (2021). Riscos ocupacionais no trabalho agrícola e a negociação para a saúde do trabalhador rural. Revista de Enfermagem da UFSM, 11(0), 43.

Carvalho, L., \& Santos, P. (2020). A Ergonomia No Contexto Das Atividades Rurais: Uma Revisão Bibliográfica. 8, $251-269$.

Carvalho, M. P. de, Fiori, N. S., Meucci, R. D., Faria, N. M. X., \& Fassa, A. G. (2020). Dor na coluna torácica e fatores associados em fumicultores. Revista Brasileira de Saúde Ocupacional, 45. 
de Oliveira, C. M., Pereira, A. C., de Andrade, M., Soler, J. M., \& Krieger, J. E. (2008). Heritability of cardiovascular risk factors in a Brazilian population: Baependi Heart Study. BMC Medical Genetics, 9, 32. https://doi.org/10.1186/1471-2350-9-32

Dias, N. T. C. (2020). Avaliação do perfil de saúde de trabalhadores rurais do município de Muzambinho - Minas Gerais.

Duarte, K. K. da S., \& Cavaignac, A. L. de oliveira. (2019). Incidência de sintomas musculoesqueléticos em trabalhadores da construção civil: estudo comparativo por funções com questionário nórdico e diagrama de corlett. Brazilian Journal of Production Engineering - BJPE, 20 -37.

Klatsky, A. L. (2016). Alcohol and cardiovascular diseases: Where do we stand today? Journal of Internal Medicine, 278(3), 238-250.

LOPES, S. A. P (2017). Prevalência de DORT em trabalhadores do setor de metalurgia cadastrados no centro de referência em saúde do trabalhador de Piracicaba/SP, no período de 2008 a 2015.

Maia, A. I. (2020). Análise de riscos e perigos relacionados a extração de carvão mineral: Medidas de controle utilizando a NR-22.

Melek, T., Gonçalves, C., Areosa, J., \& Soares, V. (2017). Condições de trabalho numa mineradora: O olhar de trabalhadores e de profissionais da saúde e segurança. 13, 104-122.

Melo, C. de F., Cavalcante, A. K. S., \& Façanha, K. Q. (2019). Invisibilização do adoecimento psíquico do trabalhador: limites da integralidade na rede de atenção à saúde. Trabalho, Educação e Saúde, 17.

MeloG. S. de O., BarbosaR. L., BarbosaF. L., BarbosaD. G., CastroM. M. da S., SilvaM. A. C. N. da, LimaM. F., SandrinP. P. de A., NascimentoM. do D. S. B., \& BarbosaM. do C. L. (2020). Perfil alimentar e nutricional da população residente em comunidades quilombolas em um município do estado do Maranhão. Revista Eletrônica Acervo Saúde, 12(1), e2957. https://doi.org/10.25248/reas.e2957.2020

Mendes, M. S. F., Alberto, N. S. M. da C., Rocha, A. V. S. G., Aguiar, A. V. G., Lima, M. E. R. de, \& Pereira, T. G. (2019). Dados sobre as condições de trabalho, perfil de saúde e nutrição de agricultores familiares de Teresina - PI, Brasil. Revista Eletrônica Competências Digitais para Agricultura Familiar, $5(2), 144-177$.

Miranda, S. V. C. de, Duraes, P. S., \& Vasconcellos, L. C. F. de. (2020). A visão do homem trabalhador rural norte-mineiro sobre o cuidado em saúde no contexto da atenção primária à saúde. Ciência \& Saúde Coletiva, 25, 1519-1528.

Minette, L. J., Schettino, S., Souza, A. P., Soranso, D. R., \& Barbosa, V. A. (2018). Colheita De Madeira Danificada Pelo Vento: Carga Física De Trabalho E Risco De Ler/Dort Aos Trabalhadores. Nativa, 6(1), 66-72. https://doi.org/10.31413/nativa.v6i1.4312

Moita, M., Ximenes Neto, F. R., Silva, R., Prado, J., Viana, T., \& Borges, G. (2020). Riscos à saúde do trabalhador rural na produção de rapadura. Research, Society and Development, $9,167953259$.

Paula, E. A. de, \& Amaral, R. M. M. F. do. (2019). Atuação interdisciplinar em grupos de qualidade de vida para pacientes com Lesões por esforços repetitivos/Distúrbios osteomusculares relacionados ao trabalho-LER/DORT. Revista Brasileira de Saúde Ocupacional, 44.

Rodrigues, B. S., Freitas, M., Tomé, D., \& Neto, H. V. (2020). Avaliação de Fadiga Laboral e Lesões Músculo-Esqueléticas Relacionadas com o Trabalho numa Secção de Mistura de Cortiça. Cesqua - Cadernos de Engenharia de Segurança, Qualidade e Ambiente, 1(3), $149-177$.

Santos, P. V. S. (2018). O papel da ergonomia em meio as atividades do campo: um olhar para o caso do vale do São Francisco. Brazilian Journal of Production Engineering - BJPE, 77-93.

Schettino, S., Guimarães, N. V., Silva, D. L. da, Souza, C. L. L. de, Minette, L. J., Junior, J. D. de P., \& Schettino, C. F. (2020). Relação entre a ocorrência de acidentes de trabalho e a baixa escolaridade dos trabalhadores no setor florestal. Brazilian Journal of Development, 6(4), 22567-22589.

Silva, I., Xavier, D., Bonow, C., Camponogara, S., Oliveira, A., Santos da Silva, M., \& Cezar-Vaz, M. R. (2020). Percepção de agricultores de soja e arroz acerca dos riscos ocupacionais. Research, Society and Development, 9, e739119543.

Silva, L. L. da, Neta, A. A. P., Prates, C. F., Soares, J. S., Araújo, T. A., Costa, A. M. A., Cardoso, T. V., \& Moura, R. C. (2021). Análise da prevalência de dor lombar associada à atividades ocupacionais: Uma revisão integrativa de literatura. Brazilian Journal of Development, 7(2), 11729-11743.

Silva, M. J. D. S., Schraiber, L. B., \& Mota, A. (2019). O conceito de saúde na Saúde Coletiva: contribuições a partir da crítica social e histórica da produção científica. Physis: Revista de Saúde Coletiva, 29.

Silva, N. R. da, Junqui, P. F., \& Silva, M. L. da. (2020). Caracterização do perfil de adoecimento por LER/DORT em um centro de referência em saúde do trabalhador. Revista Interinstitucional Brasileira de Terapia Ocupacional - REVISBRATO, 4(4), 631-645.

Silveira, J., Cezar-Vaz, M. R., \& Xavier, D. (2020). Análise ergonômica e de alterações osteomusculares em trabalhadores: Revisão integrativa. Research, Society and Development, 9, e349119500.

Simas, J. M. M., Alencar, M. do C. B. de, \& Yamauchi, L. Y. (2020). Distúrbios osteomusculares em trabalhadores da bananicultura. BrJP, 3 , $33-36$.

Souza, S., Amorim, R., Lima, M., Vale, P., Robledo, S., Rios, A., Araújo, N., \& Jesus, I. (2021). Percepção Das Práticas Ergonômicas Entre Carregadores De Uma Feira-Livre Em Feira De Santana, Bahia. Brazilian Journal of Development, 7, 3170-3190.

TRABALHO, Organização Internacional do (2002). C176 - Convenção sobre segurança e saúde nas minas. https://www.ilo.org/brasilia/convencoes/WCMS_236694/lang--pt/index.htm. Acesso em: 13 jan. 2021.

Vale, S. R. G. A., Bonfatti, R. J., Souza, A. G., \& Teixeira, L. R. (2018). Análise ergonômica da atividade de quebra tradicional do coco babaçu no município de Itapecuru-Mirim/MA. Revista Brasileira de Saúde Ocupacional, 43. 
Veiga, J. P. C., Trevisani, D. M., Makishi, F., Abreu, M. G. C. de, Silva, M. S. P. e, \& Zacareli, M. A. (2017). Padrões de saúde e segurança no trabalho e extrativismo: O caso de comunidades rurais da Amazônia brasileira. Saúde e Sociedade, 26, 774-785.

Victorio, V. C. M., \& Gonçalves, E. C. B. de A. (2021). Aspectos de saúde relacionados a idade, escolaridade e produções agrícolas de agrcultores no Assentamento Rural Rio Madeira-RO. Agricultura Familiar: Pesquisa, Formação e Desenvolvimento, 14(2), 183-202.

Ximenes Neto, F. R., Oliveira, G., dos Santos, F., Sousa, F., Vasconcelos, L., Ferreira, V., \& Lourenção, L. (2020). Accidents with workers and rural workers: From the case series to the evolution of cases. Research, Society and Development, 9, 191973949.

Zavarizzi, C. de P., Carvalho, R. M. M. de, \& Alencar, M. do C. B. de. (2019). Grupos de trabalhadores acometidos por LER/DORT: Relato de experiência. Cadernos Brasileiros de Terapia Ocupacional, 27, 663-670. 